\title{
FRECUENCIA DE INFECCIÓN CON ACTINOBACILLUS PLEUROPNEUMONIAE EN GRANJAS PORCINAS TECNIFICADAS DE LA COSTA PERUANA
}

\section{Frequency of ACtinobacillus PlEURopneumoniae INFECTION IN CoMmerCial Pig Farms of the Peruvian Coast}

\author{
Lorena Mori A. ${ }^{1}$, Sonia Calle E. ${ }^{1,2}$, Chris Pinto J. ${ }^{1}$, Marlon Torres A. ${ }^{3}$, \\ Néstor Falcón P. ${ }^{4,5}$, Siever Morales C. ${ }^{1}$
}

\section{RESUMEN}

\begin{abstract}
El objetivo del estudio fue determinar la frecuencia de anticuerpos contra la toxina ApxIV de Actinobacillus pleuropneumoniae, causante de pleuroneumonía porcina en 10 granjas porcinas tecnificadas de los departamentos de Arequipa, Lima, Ica y La Libertad. Se colectaron muestras de sangre de porcinos de las etapas de crecimiento y acabado (30 por granja) y se analizaron mediante la prueba de ELISA indirecta con un kit comercial. El 23.7\% (71/300) de los animales presentaron anticuerpos contra la toxina ApxIV, correspondiendo la mayor frecuencia a la granja del departamento de Ica (60.0\%). La presencia de anticuerpos contra la toxina ApxIV evidencia la presencia de la infección con A. pleuropneumoniae en la industria porcina del Perú.
\end{abstract}

Palabras clave: Actinobacillus pleuropneumoniae, pleuroneumonía porcina, toxina Apx IV, ELISA indirecta

\section{Abstract}

The objective of the study was to determine the frequency of antibodies against the ApxIV toxin of Actinobacillus pleuropneumoniae, causal agent of porcine pleuropneumonia in 10 commercial pig farms of Arequipa, Lima, Ica and La Libertad departments. Blood samples were collected from animals in the growing and finishing stages (30 per farm) and analyzed by the indirect ELISA test using a commercial kit. The results showed that $23.7 \%$ (71/300) of the animals presented antibodies against the ApxIV toxin, and the highest frequency was found in the farm located in Ica (60\%). The presence of antibodies against the ApxIV toxin indicated the presence of A. pleuropneumoniae infection in pig commercial farms in Peru.

Key words: Actinobacillus pleuropneumoniae, porcine pleuropneumonia, ApxIV toxin, indirect ELISA

${ }^{1}$ Laboratorio de Microbiología y Parasitología Veterinaria, ${ }^{3}$ Laboratorio de Zootecnia y Producción Agropecuaria, ${ }^{4}$ Laboratorio de Medicina Veterinaria Preventiva, Facultad de Medicina Veterinaria, Universidad Nacional Mayor de San Marcos, Lima

${ }^{2}$ E-mail: calleson@gmail.com

${ }^{5}$ Dirección actual: Facultad de Veterinaria y Zootecnia, Universidad Peruana Cayetano Heredia, Lima 
La industria porcina se ve afectada por problemas de origen sanitario, siendo uno de los principales, las enfermedades de tipo respiratorio, vistas en la forma de neumonía y pleuritis, ya sean de origen bacteriano o viral. La ocurrencia de estas enfermedades en las granjas porcinas afecta seriamente los indicadores de producción, pues ocasiona una disminución en la tasa de crecimiento, en la ganancia diaria de peso, en la conversión alimenticia, en el consumo de alimento y en el peso de los animales al mercado.

La pleuroneumonía porcina producida por Actinobacillus pleuropneumoniae es una de las enfermedades de mayor impacto económico asociadas al complejo respiratorio porcino, debido principalmente a la alta mortalidad, costos de medicación y disminución en la tasa de crecimiento. El gran intercambio comercial, la intensificación de la producción y la masificación en las explotaciones porcinas predisponen la diseminación de esta enfermedad, incrementando las pérdidas económicas.

En el Perú, Calle y Camacho, en 1988, aislaron por primera vez A. pleuropneumoniae de muestras de cerdos en el departamento de Trujillo (S. Calle, datos no publicados). Posteriormente, a través de estudios serológicos en la zona de Lima se detectaron anticuerpos contra los serotipos 1, 3, 5, y 7 de la bacteria, usando la prueba de aglutinación en placa, confirmando de esta manera la presencia de A. pleuropneumoniae en las granjas porcinas tecnificadas del Perú (Calle et al., 1996; Castillo, 1996).

El presente estudio tuvo por objetivo determinar la frecuencia de infección con $A$. pleuropneumoniae en granjas porcinas tecnificadas de Arequipa, Lima, Ica y La Libertad.
El estudio se realizó entre los años 2005 y 2007 en 10 granjas porcinas tecnificadas, inscritas en la Asociación Peruana de Porcicultores, y que se encontraban ubicadas en los departamentos de Arequipa (1), Ica (1), Lima (Lima Norte [4], Lima Sur [1], Lima Este [1] y Lima Oeste [1]) y La Libertad (1). El número de madres en estas granjas varió entre 150 y 3500, siendo la mediana de 600 madres.

Para el cálculo del tamaño muestral se utilizó el Teorema del Límite Central, requiriéndose 30 muestras por granja como mínimo, las cuales fueron seleccionadas al azar y distribuidas en 2 grupos según etapa productiva: gorrinos en crecimiento (entre 9 a 17 semanas) y en acabado (entre 18 a 22 semanas).

Se colectaron muestras de sangre mediante punción de la vena cava anterior. El suero se obtuvo por centrifugación a 3500 rpm durante cinco minutos y fue conservado a $-20{ }^{\circ} \mathrm{C}$ hasta su procesamiento en el Laboratorio de Bacteriología de la Facultad de Medicina Veterinaria (FMV) de la Universidad Nacional Mayor de San Marcos (UNMSM).

Los sueros fueron analizados mediante la prueba de ELISA (Enzyme Linked Immunosorbent Assay) empleando el Kit CHEKIT APP-ApxIV (IDEXX Laboratories). El kit detecta anticuerpos contra la toxina ApxIV que es específica de $A$. pleuropneumoniae pero común a todos los serotipos. La prueba tiene una sensibilidad del 95\% y una especificidad del 99\%. La lectura de la absorbancia de cada pozo de realizó en un lector de microplacas (Bio-tek ELx800) a una longitud de onda de $450 \mathrm{~nm}$. A través de los valores de densidad óptica (DO) se calculó los valores de las muestras 
Cuadro 1. Porcentaje de muestras seropositivas a Actinobacillus pleuropneumoniae en cerdos de granjas tecnificadas ( $\mathrm{n}=30$ animales por granja)

\begin{tabular}{lccc}
\hline \multirow{2}{*}{ Zona } & Granja & $\mathrm{n}$ & \multicolumn{2}{c}{ Animales positivos } \\
\cline { 3 - 4 } & & 13 & 43.3 \\
& 1 & 1 & 3.3 \\
Lima Norte & 2 & 10 & 33.3 \\
& 3 & 1 & 3.3 \\
Lima Sur & 4 & 5 & 16.7 \\
Lima Este & 5 & 1 & 3.3 \\
Lima Oeste & 6 & 10 & 33.3 \\
Ica & 7 & 18 & 60.0 \\
La Libertad & 8 & 2 & 6.7 \\
Arequipa & 9 & 10 & 33.3 \\
\hline Total & 10 & 71 & 23.7 \\
\hline
\end{tabular}

en relación al valor de DO del control positivo y DO del control negativo.

Se determinó la frecuencia de muestras seropositivas a A. pleuropneumoniae del total de muestras y según las variables zona geográfica y etapa productiva. Para el análisis de la asociación de estas variables y la frecuencia de muestras seropositivas se utilizó la prueba de Chi Cuadrado utilizando el paquete estadístico SPSS.

\section{Resultados y Discusión}

Se detectó la presencia de anticuerpos contra la toxina ApxIV de $A$. pleuropneumoniae en el $23.7 \%$ de los gorrinos muestreados (Cuadro 1). Esta toxina se produce durante la infección activa, lo que significaría que los animales detectados como positivos estarían infectados con este patógeno.
Con la intensificación de los sistemas de producción porcina se incrementa la densidad de animales por corral y el ingreso de animales de reemplazo y, por lo tanto, aumenta el riesgo de enfermedades. En la mayoría de los casos, las granjas porcinas comerciales de la zona no realizan pruebas diagnósticas para el descarte de $A$. pleuropneumoniae en los animales de reemplazo, y este es uno de los factores claves para la introducción del agente a una granja (Wongnarkpet et al., 1999). Además, el A. pleuropneumoniae ya se había detectado en granjas comerciales del país (Castillo, 1996), por lo que la presencia de animales seropositivos era esperado; sin embargo, los valores encontrados son bastante bajos en relación a los reportes de Castillo (1996) en Lima, con 76.5\%, utilizando la técnica de aglutinación en placa.

Estudios realizados en otros países han reportado valores mayores al encontrado en 
este estudio, como es el caso de Brasil con 48.3\% (Moreno et al., 1999) con la prueba de inhibición de la hemolisina, México con 96.6\% (Moguel, 1997) y 100\% (Rodríguez, 2004) con la prueba de aglutinación en placa, EEUU con un 32.1\% (Schultz et al., 1982) utilizando la prueba de fijación de complemento, y Turquía (Metiner y Ak, 2007) con un $67.2 \%$ con un ELISA basado en la toxina ApxI y la proteína de membrana externa Tbp2. La prueba de inhibición de la hemolisina y el ELISA basado en la toxina ApxI y Tbp2 no son capaces de diferenciar las infecciones producidas por otras especies bacterianas que producen toxinas y proteínas similares, como E. coli hemolítica, A. suis y $H$. parasuis. Igualmente, la prueba de fijación de complemento y la prueba de aglutinación en placa pueden dar resultados falsos positivos (Medrano, 2003; Gottschalk, 2004).

Las diferencias en resultados de los monitoreos serológicos realizados en granjas porcinas para determinar la frecuencia de animales seropositivos a A. pleruropneumoniae puede deberse a la sensibilidad y especificidad de las pruebas usadas, el origen de los animales, el sistema de crianza y el medio ambiente. Sin embargo, lo más importante por considerar, es la propia dinámica de la infección que depende del manejo de los animales y de la posible introducción de animales con infección subclínica en cada granja (G. Zielinski, datos no publicados).

La granja de la zona de Ica mostró el mayor porcentaje de seropositividad (60\%, Cuadro 1). Todas las granjas realizan un manejo similar de los animales; sin embargo, en granjas multi-sitios, como el caso de la granja de Ica, se realiza un constante traslado y movimiento de animales, así como la reagrupación de animales según edad, peso o tamaño en las etapas de recría, crecimiento y acabado. Este tipo de manejo permite un mayor contacto directo entre animales infectados y susceptibles, aumentando el riesgo de infección. Un estudio reciente detectó la infección con A. pleuropneumoniae en el 94.1\% de madres de una granja en Ica (García,
2007), estimándose que los lechones podrían infectarse durante la etapa de lactación. En consecuencia, al realizar un agrupamiento de estos animales en la etapa de recría, se facilitaría la transmisión del agente de los animales infectados a los susceptibles. Vigre et al. (2002) demostraron que la seroconversión ocurre entre las 2 a 4 semanas post infección, de allí que los animales que se infecten en la etapa de recría (4 a 7 semanas de edad) comenzarían a presentar anticuerpos entre las 6 y 8 semanas de edad.

En base a esta información y los hallazgos de Haesebrouck et al. (1997), que encontraron el nivel máximo de anticuerpos entre las 3 a 4 semanas post infección, pudiendo perdurar estos anticuerpos por varios meses, se podría entonces, encontrar altos niveles de anticuerpos debida a una infección en los animales en la etapa de crecimiento. En el estudio se encontró una frecuencia de 28.5\% (55/193) en los gorrinos de crecimiento y $15.0 \%$ (16/107) en los de acabado. El menor valor en estos últimos se debe a que la seroconversión a $A$. pleuropneumoniae depende del momento en que ocurre la infección que, a su vez, está sujeto al comportamiento del agente y al manejo en cada granja.

Los anticuerpos de origen maternal pueden perdurar hasta por 12 semanas, de modo que debido al diseño del estudio y la prueba de ELISA usada, no se puede diferenciar los anticuerpos de origen maternal de aquellos productos de una infección. Por esto, no se podría afirmar que la seropositividad encontrada en los gorrinos a partir de los 60 días de edad sea en su totalidad debida a una infección activa. En todo caso, se podría estar frente a una fuerte transferencia de la inmunidad pasiva (García, 2007). No obstante, Haesebrouck et al. (1997) señalan que la protección de los anticuerpos maternales no supera las 3 semanas; además, se reconoce que la prueba de ELISA indirecta detecta generalmente anticuerpos a partir de las 3 semanas post infección (Dreyfus et al., 2004) y, por último, se ha determinado que 
los animales se tornan susceptibles a la infección a partir de las 8 semanas de vida (Chiers et al., 2002), de allí que se podría sugerir que la seropositividad encontrada a partir de los 60 días de edad puede ser consecuencia de una probable infección activa.

\section{Conclusiones}

- Las 10 granjas fueron seropositivas a Actinobacillus pleuropneumoniae, encontrándose una mayor frecuencia de animales seropositivos en la zona de Ica (60\%).

- Se encontró una mayor frecuencia de animales seropositivos (28.5\%) en la etapa de crecimiento que en la etapa de acabado (15.0\%), sugiriendo que esta etapa es la de mayor riesgo de infección.

\section{Literatura Citada}

1. Álvarez $M$, Rodríguez J, Ciprian A, Rodríguez L, Ayora G Segura J. 2004. Perfil serológico del virus de influenza porcina, Micoplasma hyopneumoniae y Actinobacillus pleuropneumoniae, en granjas de Yucatán, México. Vet Mex 35: 295-305.

2. Calle S, Camacho C, Urtega L. 1996. Detección de anticuerpos contra Actinobacillus pleuropneumoniae en granjas porcinas en Lima. En: XIII Congreso Nacional de Ciencias Veterinarias. Lima. p 231.

3. Castillo C. 1996. Aislamiento y determinación de serotipos de Actinobacillus pleuropneumoniae en porcinos beneficiados en camal de Lima. Tesis de Médico Veterinario. Chiclayo: Facultad de Medicina Veterinaria, Univ Nac Pedro Ruiz Gallo. 38 p.

4. Chiers K, Donné E, Van Overbeke I, Ducateller R, Haesebrouck F. 2002. Actinobacillus pleuropneumoniae infections in closed swine herds: infection patterns and serological profiles. Vet Microbiol 85: 343-352.

5. Dreyfus A, Schaller A, Nivollet S, Segers RPAM, Kobisch M, Mieli L, Soerensen, et al. 2004. Use of recombinant ApxIV in serodiagnosis of Actinobacillus pleuropneumoniae infections, develop-ment and prevalidation of the ApxIV ELISA. Vet Microbiol 99: 227-238.

6. García O. 2007. Persistencia de la inmunidad pasiva contra Actinobacillus pleuropneumoniae en porcinos en etapa de recría. Tesis de Médico Veterinario. Lima: Facultad de Medicina Veterinaria, Univ Nac Mayor de San Marcos. 64 p.

7. Gottschalk M. 2004. Avances recientes en el diagnóstico y el control de la pleuropneumonía porcina. Mundo Ganadero. 170. [Internet]. Disponible: http:// www.exopol.com/general/circulares/ 103circ.html

8. Haesebrouck F, Chiers K, Van Overbeke I, Ducatelle R. 1997. Actinobacillus pleuropneumoniae infections in pigs: the role of virulence factors in pathogenesis and protection. Vet Microbiol 58: 239-249.

9. Medrano A. 2003. Expresión recombinante en $E$. coli de antígenos de Actinobacillus pleuropneumoniae para vacunación y diagnóstico. Tesis de Doctorado. Barcelona, España: Instituto de Biotecnología y Biomedicina, Universidad Autónoma de Barcelona.

10. Metiner K, Ak S. 2007. Presence and seroprevalence of Actinobacillus pleuropneumoniae in pigs in Turkey. Acta Vet Brno 76: 237-244.

11. Moguel JR. 1997. Seroprevalencia y descripción de algunas variables relacionadas a la presentación de Actinobacillus pleuropneumoniae en granjas porcinas de Yucatán. Tesis de Maestría. Yucatán: Facultad de Medicina Veterinaria y Zootecnia, Univ Autónoma de Yucatán. 67 p.

12. Moreno AM, Barbarini O, Baccaro MR. 1999. Levantamento sorológico para Actinobacillus pleuropneumoniae em 
criações de suínos no período de dezembro de 1996 a julho de 1999. En: IX Congresso Brasileiro de Veterinários Especialistas em Suínos. Belo Horizonte.

13. Schultz RA, Young TA, Ross DF, Jeske DR. 1982. Prevalence of antibodies to Haemophilus pleuropneumoniae en Iowa swine. Am J Vet Res 43: 1848-1851.

14. Vigre H, Oystein A, Barfod K, Lavritsen D, Sorensen V. 2002. Transmission of Actinobacillus pleuropneumoniae in pigs under fieldlike conditions: emphasis on tonsilar colonization and passively acquired colostral antibodies. Vet Microbiol 89: 151-159.

15. Wongnarkpet S, Pfeiffer DU, Morris RS, Fenwick SG. 1999. An on-farm study of the epidemiology of Actinobacillus pleuropneumoniae infection in pigs as part of a vaccine efficacy trial. Prev Vet Med 39: 1-11. 